\title{
Pediatricians Do Not Ask Routinely About Parental Smoking Habits
}

\author{
Wojciech Feleszko, ${ }^{1,}$ Aleksandra Rurarz, ${ }^{1}$ and Karol Ratajczak ${ }^{1}$ \\ ${ }^{1}$ Department of Pediatric Pulmonology and Allergy, Medical University of Warsaw, Warsaw, Poland \\ "Corresponding author: Wojciech Feleszko, MD, PhD, Department of Pediatric Pulmonology and Allergy, Medical University of Warsaw, ul. Zwirki i Wigury 63A, 02-091, Warsaw, \\ Poland. Tel: +48-223179419, E-mail: wojciech.feleszko@wum.edu.pl
}

Received 2016 December 09; Revised 2016 December 28; Accepted 2017 February 11.

Keywords: Parental Smoking, Children, Tobacco Smoke

\section{Dear Editor,}

The Journal recently published a study (1) on the current data concerning exposure to second hand smoking among Iranian children and adolescents. In particular, the authors noted low parental education correlating with increased tobacco smoke exposure (TSE) frequency among children and pointed out a need to improve the effectiveness of family-centered counseling as well as legislation for smoke free home.

Passive smoking is consequently demonstrated to be causally associated with a large number of human diseases although in the view of many authors the obvious association between parental smoking and recurrent respiratory infections or asthma seems to be ignored by many pediatricians and general practitioners (2). Nevertheless, vast majority of parents (89\%) claim that asking about parental smoking status is an important part of pediatrician's role (3).

In our recent analysis, 77\% (392 out of 506) of parents whose children hospitalized due to respiratory problems have never been asked about smoking habits during routine pediatric consultation (data in preparation). Interestingly, this tendency significantly reduced among those whose health care service was performed by GPs (59\%). A strong discrepancy between different cultures/nations seems to exist since the proportion of pediatricians who routinely do not ask about environmental tobacco smoke in the US and Israel was $27 \%$ and $43 \%$, respectively $(4,5)$. However, these data still indicate relatively low rates of screening and counseling for parental smoking in pediatric practices while regrettably, among these families many parents (40\%) report regular smoking habits.

As reported by our investigation, rule banning smoking in the car, in which a child travels, is valid for only $23 \%$ of Polish families. This value is surprisingly low compared to other countries (data in preparation). In Australia, up to $96 \%$ of respondents claim that they prohibit smoking in the car (6). In Wales, only $9 \%$ of the respondents agree for smoking inside the vehicle which is used by the child (7). It is therefore crucial that pediatricians and general practitioners suggest quitting smoking in vehicles used by children.

According to our survey, smoking parents try to quit smoking to improve their child's health and such attempts were reported by 4 out of 10 smoking parents.

We do agree with Kelishadi and colleagues that the current public health education methods of anti-smoking interventions are inadequate maybe due to the lack of attractive smoking cessation tools. E-cigarettes seem to be an interesting alternative due to tremendous public interest and current data suggesting that even though they are not completely neutral for human health, they still are less harmful to children than conventional cigarettes (8). 

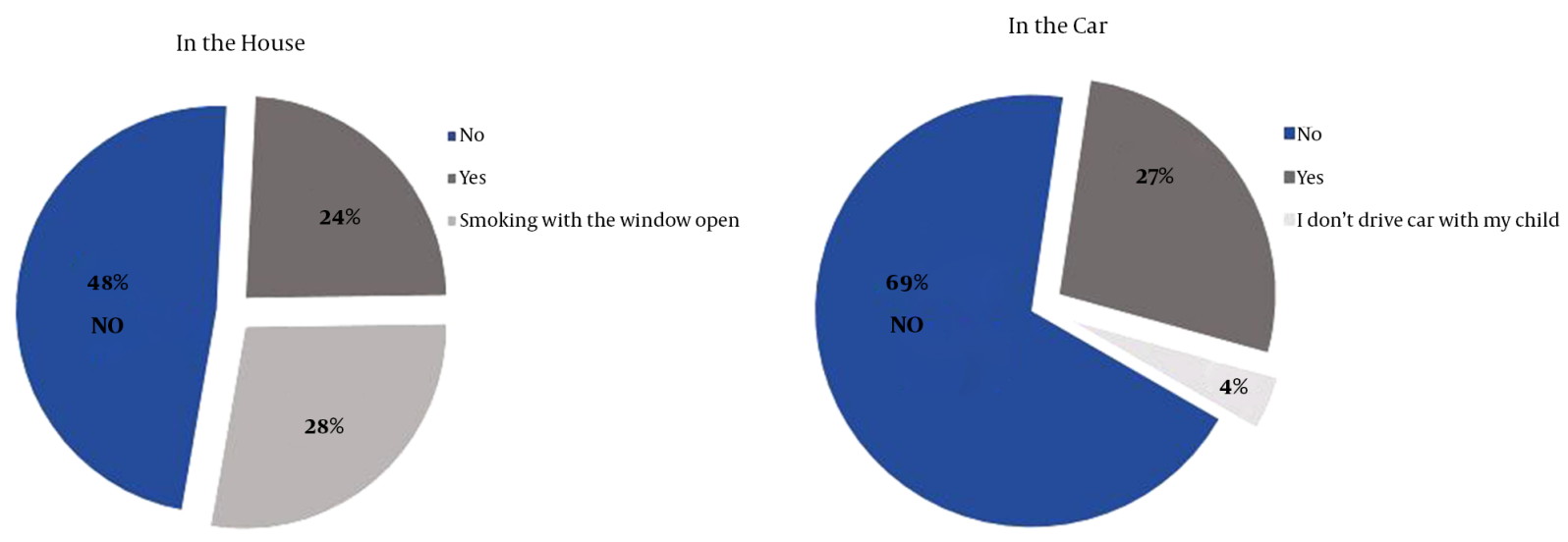

Figure 1. Percentage of Parents Having a Rule Banning Smoking

\section{References}

1. Kelishadi R, Shahsanai A, Qorbani M, Ardalan G, Poursafa P, Heshmat R, et al. Exposure to Hookah and Cigarette Smoke in Children and Adolescents According to Their Socio-Economic Status: The CASPIAN-IV Study. Iran J Pediatr. 2016;26(4):e3036. doi: 10.5812/ijp.3036. [PubMed: 27781078].

2. Daly JB, Mackenzie LJ, Freund M, Wolfenden L, Roseby R, Wiggers JH. Interventions by Health Care Professionals Who Provide Routine Child Health Care to Reduce Tobacco Smoke Exposure in Children: A Review and Meta-analysis. JAMA Pediatr. 2016;170(2):138-47. doi: 10.1001/jamapediatrics.2015.3342. [PubMed: 26719991].

3. Moss D, Cluss PA, Mesiano M, Kip KE. Accessing adult smokers in the pediatric setting: What do parents think?. Nicotine Tob Res. 2006;8(1):67-75. doi: 10.1080/14622200500431809. [PubMed: 16497601].

4. Sharif I, Oruwariye T, Waldman G, Ozuah PO. Smoking cessation counseling by pediatricians in an inner-city setting.J Natl Med Assoc. 2002;94(9):841-5. [PubMed: 12392048].

5. Rosen L, Kostjukovsky I. Parental risk perceptions of child exposure to tobacco smoke. BMC Public Health. 2015;15:90. doi: 10.1186/s12889-015-1434-x. [PubMed: 25885053].

6. Glover M, Kira A, Johnston V, Walker N, Brown N, Thomas D. Australian and New Zealand Indigenous mothers' report respect for smoking bans in homes. Women Birth. 2015;28(1):1-7. doi: 10.1016/j.wombi.2014.09.004. [PubMed: 25458756].

7. Moore GF, Moore L, Littlecott HJ, Ahmed N, Lewis S, Sulley G, et al. Prevalence of smoking restrictions and child exposure to secondhand smoke in cars and homes: a repeated cross-sectional survey of children aged 10-11 years in Wales. BMJ Open. 2015;5(1):e006914. doi: 10.1136/bmjopen-2014-006914. [PubMed: 25636793].

8. Wasowicz A, Feleszko W, Goniewicz ML. E-Cigarette use among children and young people: the need for regulation. ExpertRev RespirMed. 2015;9(5):5079. doi: 10.1586/17476348.2015.1077120. [PubMed: 26290119]. 